\title{
Namenregister
}

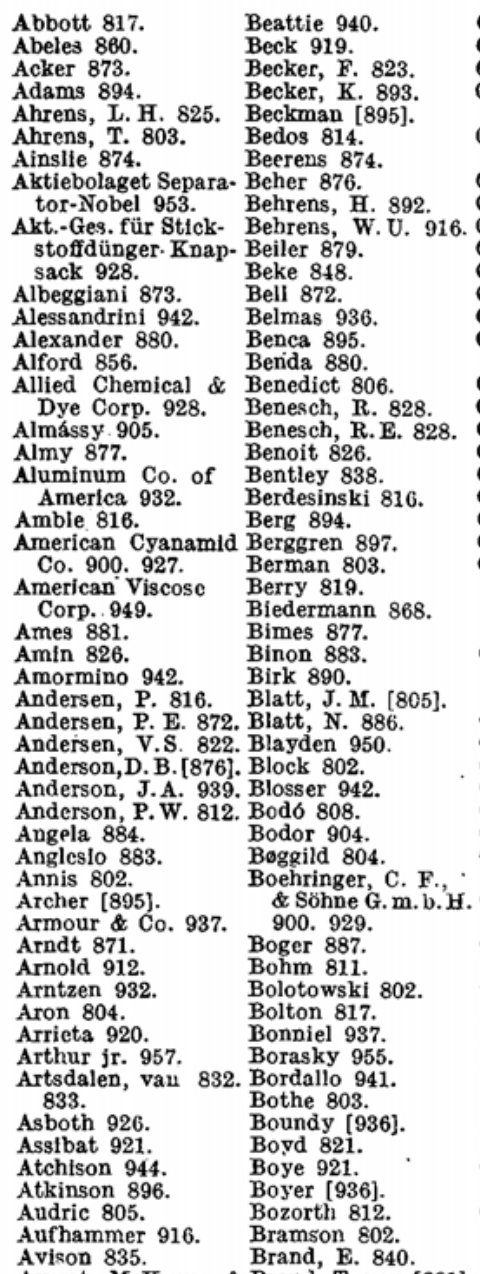

Avison 835 . Brand, E. 840

Harrison Ltd. 901. Brandenburger 947 .

Baar 825

Bach [881].

Bacher 885 .

Bachman 901

901.

Soda-Fabrik 912 Breuer 897.

913. 923. 926. 927. Brewer 887.

944. 948, 953. 954. Bridge 802.

Bächli 860 .

Baffi 889 .

Baguena Candela Ltd. 938.

883.

Bahlo 957.

Bailitis 814 .

Balzer 862.

Brandes 897 .

Brandstätter 897

Calingaert 828

Dean, H. 827.

Calleja 914.

Dean, W. T. 923

Callen 809.

Dechamps 883.

Canadian Kodak Co., Dehn 912

Ltd. 959.

Dekker, K. D. 938

Westing

Dekker, M. J. D.

house Co. 933.

Capalbi 885.

Cardwell 831. 832.

938.

Delavierre 891.

Carentoni 959

Carson 873

Carter, H. E. 867.

Carter, T. J. 956.

Casillas 888.

De Lollis 816.

Demeter 942.

Desai 853. 854.

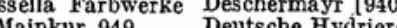

Dainkur 949. Deutsche Hydrier-
werke Akt.-Ges.

Castillo, Del 802 . 933.

Cattaneo 873. 883. Deutsches Hydrier-

Chalatnikow 813 .

Chalmers 812.

Chang, W. Y. 802.

Chang, Y. L. 830 .

Chasson 801 .

Chemische Verwer-

tungsgesellschaft

Oberhausen

m. b. H. 927 .

emische Werke

Hüls G.m.b.H.

926. 934.

Chen 875.

werk Rodleben

VEB 898. 935 .

936.

.

Deysson 871 .

Dharmatti 813.

Diamond Alkali Co. 912. 926

Diaz 867.

Dibeler 827 .

Dietrich 939.

Dijkstra 812

Dikhoff 808 .

Dintilhac 908.

Chiurdoglu 830. 831. Distíllation Products

Christenson 911.

Inc. 900.

Christman jr. 814 jerassi 862. 864

Ciba Akt.-Ges. 900. Dobrotworskaja 930.931. 933, 957. Dobrovolskaia-

Ciba Pharmaceutical Zavadskaĩa 889

Products, Inc. 899. Doemens [940].

Civeira 892 .

Doering 868 .

Clar 850.

Doležálek 941.

Cleveland 808 .

Cochran 817 .

Cohen 832. 833.

Donner 878 .

Dorfman 860 .

Dow Corning Corp.

Cohn 879 . 944.

844. 847.

Collins 940 .

raczynski 940.

Comar 880

Drummond 806.

Valentine 932 .

Dubois, E, 923 .

Comptoirdes Textiles Du Bois, $\mathbf{K}$.

(S.à. R. I

Compton 876.

Dudley 917.

Duke, E. T. 911.

Duke, F. R. 822

Dullien 907 .

Dunn 859.

Conroy 868

Cooil 875 .

Cook 907 .

Du Por

Pont de Nemours Gaes

Co. 925. Gaetani, de 886 .

937. 950. Galactina \& Biom

Corning Glass Works Durham 951. 914.

Duval 905 .

Coulson 817.

Duyckaerts 903.

Courtaulds Ltd. 946. Dyer 880 .

949.
Cox 837. 838. 839. Eastman Kodak Co. Geigy, J. R., Akt.-

allup 880

Garaventa 884.

Garrod 886.

Gasser 917.

949.
Cox 837. 838. 839. Eastman Kodak Co. Geigy, J. R., Akt.-

Crabtree 934. 946. 959.

Cramer 907.

Crecelius 880

Broeg 938 .

Baker Platinum Ltd. Broser, I. 960.

924.

Broser, W. 868.

Ballerini 882 .

Ballczo 904.

Banerjee 941.

Barber 891.

Barer 907.

Bargeton 891

Brown, J. M. 911.

Corp. 912.

Barnard 922.

Barone 814.

Barrow 806.

Bartholomew 887 .

Barton 864 .

Bashenow 869 .

Basolo 828 .

Bass 939.

Batres 862
Crescitelli 889 .

Bronson 917.

therton \& Co. Ltd. 912

Brown, A. H. 871.

Bru 877.

Cristol 833

\& Industries Ltd.

Cullison 880

Cunha, da $87 \pi$.

Curie 803 .

Curtin 880

Curtis 801 .

Czech 914.

Dale 872 .

Daniels 886 .

Danzig 851

Davey 879 .

Davis, A. C. 852 .

Burgio 873 .

Burke 888 .

Burt 828.

Butler 887.

Buru-Hol 883 .

Dawes 872 .

Dayton'Rubber Co. Erdey 904. 937 .
Electric \& Musical
Eastwocd 952 .

Eberhardt 919 .

Eden 911 .

Edwards 809 .

Eitel [814]. 910.

Fllner 862 .

Elvehjem 880 .

Emeléus 824.

Emerson \& Cuming Co. 927.

Emigh 803.

Emmett 823.

Enderby 891

Engel 880.

Engell 921.

Erb 942

Erbslöh 890 .

Ges. 948.

General Aniline \& Film Corp. 960

General Electric Co. Ltd. 913.

eneral Mills, Inc. 928.

Génin 934.

Gentieu 921.

Gentry 905.

Gerds 824.

Erlanger 840 .

G.m.b.H.

Gitzen 24.

Glover 194.

Goates 822 .

Goddard 871 .

Görges 881 .

Goetz 889 .

Gohr 881

Goldberg 893 .

870 .

Goldschmidt, Th.

Akt.-Ges. 913.

918. 935

Gora 802

Gordon, M. 937.

Gorman 911 .

Gould 917.

Govindan 875 .

Gow 812.

Grady 920 .

Graefe 941.

Graf 878.

Grashdanzew 920 .

Graßmann 956.

Grassner 906.

Gray 893.

Gregg jr. 804

Grob 917.

Grodzins 802.

Grü mann G.m.b.H 915.

Grütz 885 .

Guastaila 815 .

Gulf Research \& Development Co. 954 .

Guterman 919

Gutmann 823

Gutzeit 952.

Gyenes 906 .

Haar, ter 812.

Haase 913.

Hackel 877 .

ärtel 907.

Hahne 929 .

Hale 901.

Hall 929 .

Hammel 813.

Hamstead 917.

Hangarter 893 .

Hansard 880 .

Hansen 897.

Happey 947 .

Harris, F. R. 824.

Harris, J. 832. 833 .

Harrison 880 .

Harrison Laborato-

ries, Inc. 937.

Hauffe 921.

Hause 833 .

Havens jr. 802

Hayashi 875 .

Hearn 867. 
Hoefer 879.

Kirschenbaum 840. Lübke 893.

Hofferbert 917 .

Ilare 947 .

Inc. 900 .

Hofman-Bang 822 . Kleber 940.

Hogan 957.

Honigmann 819 . Klemens 809.

Hiinlich 929 .

Hüttenwerk Rhein- Knight 828.

hausen Akt.-Ges. Knoppers 891. 922.

Hughes 952

Humphreys 806. Kobayashi 892 .

Hungermann 810. Koch, B. 903.

Hupka 892.

Hyde 947.

I. G. Farbenindustrie Körösy 903.

Akt.-Ges. 949. Kofler 819.

Ilveskorpi 933.

Imhausen 943.

Co.

G.m.b.H. 943 .

Imperial Chemical

Industries Ltd.

Industrial Accoun- Korowkin 916. tancy Partnership Koshewnikow 956. Ltd. 949.

International Elcc- Kot 938. tric Co., Inc. 923. Kovács 848 .

InternationalGeneral Krapf 917. Electric Co., Inc. Krasemann 914 933.

International Nickel Krebs, K. C. 892. Co. Inc. 925. Kreidl 933.

Irish 894.

Irmann 920

Isaacs 802 .

Ishikawa 811 .

Ito 820 .

Iwanowska 805 .

Jacobi 813.

Jaeger 914.

Jain 811

Jakab 902.

Jakobson 944

Jámbor 903 .

James 811 .

Jaworski 806.

Jenikejew 938.

Jensen 808.

Jezíc 888.

Jirásek 895 .

Kruijer 941.

Jányi 902.

Johnson, G. D. 901. Lapan 889

Johnson, H. R. 807. Laquer 813.

Johnson, M. J. 902. Largent 880 .

Johnson, V. A. 812. Latham [915].

Johnston, J. 887 . Lautsch 868.

Johnston, R. L. 879. Lazarus 877.

Jones, F. E. [915]. le Hihan 883.

Jone3, S. S. 821 . Lennert 907.

Jonge, de 932 Leonard 854.

Jongh, de 891. 893. Lepper 886 .

Jordan $880 . \quad$ Lepri 885.

Joseph 882.

aalund-Jorgensen 892.

Kimpf 916.

Kainarski 913.

Kalle \& Co., Akt. -

Ges. 936. 960.

Kalyanum 882

Kamphenkel 946.

Kanagy 956.

Kanarskaja 869.

Kantrowitz, 813.

Karelitz 886.

Karpman 801

Katzin 825 .

Kaufman 907.

Kaufmann 904.

Kearns 825 .

Keitel 912.

Kellogg, M. W., Co. 911.

Kemp 957.

Kenyon 907.

Kern jr. 877.

Keuning, de 881 .

Kilchling 883.

Kilner 831.

Kinzinger 803.

Kipp 945.

Kirchmeyer 897 .

Kirketerp 872.

Kirkwood 828. Kittel 812. Klauditz 945.

Klosa 899.

Knorr 939.

Koch, J. 959.

Kock 942.

Kofler 819 .

Kok 893.

Kolomenski 802 .

Kootz 890 .

Kooy 930 .

Kopineck 806

Koppe 811.

Kcski 875 .

Krebs, H. 818.

Kríshnan 811.

Kroeger 808.

Kuch 930.

$$
820 .
$$

Kummer 823.

Kunstzeidefabrik Bobingen 950 .

Kunzler 814.

Kutjanin 955 .

Kwasniewski 896 .

Laitinen 903.

Lambert 941.

Lang 892.

Langenberg 881 .

Lanning 936 .

Lans 881 .

Lansford jr. 867.

Leser 956.

Luecke 879 .

umpkin 829.

Lund 946 .

Lupi 884.

Lu.skin 839 .

Luttinger 812 .

Ic Carthy 911 .

Nacchi 889.

Itaccoll 817 .

Mc Collister 894

McCord 804.

McCullough 919.

McDonald 903.

McGee 813.

McGraw 913.

MacGregor 804.

McIntosh 804.

McKay 810.

MacNamee 893.

McRary 901.

McRoberts 942.

McWilliams 922 .

Maddams 827.

Maler 829.

Majorana 807 .

Mallet 872 .

Mallett 824.

Mancera 864

Mande 805 .

Manes 951 .

Mann 956.

Mapstone 951.

Maraballi 853 .

Mariani 883.

Mariller 927.

Marnon 929.

Marquès 877.

Martin, G. J. 879.

Martin, 0. 952

Martin, R. B. 945.

Martius, U. 812 .

Martius, U. M.

Masera 882 .

Mataré 810 .

Matís 891 .

Matteuch 887.

Mautner 941.

Mazzetta 884

Medical Research Council 885.

Meek 833.

Meen 859.

Mellor 957 .

Mentzer 883.

Merck \& Co., Inc. 900

Meredith 939.

Messer, Adolf, G.m.b.H. 908.

Metallgesellschaft

Akt.-Ges. 938.

943.

Mfeyer, B. S. [876].

Meyer, K. A. 881 .

Meyer-Arendt 881

Meyer-Brunot 907

Leurgans 806 .

Lever Brothers \& Meynet 921. Unilever Ltd. 943. Mez 829.

Levin 826.

Levine 877

Levy 852 .

Lidiard 813.

Lincoln 917.

Linder 947.

Lindquist 879 .

Lindsey 901.

Line jr. 834 .

Link 878.

Linstedt 816

Lipson 817.

Lister 929 .

Llewellin 894 .

Löhr 890 .

Long, F. A. 821.

Long, J. V.P. 805.

Loos 816 .

Loosli 880 .

Loraine 876 .

Lord 903

Loter 862 .

Louda 934 .

Loughlin 882 .

Lowden 911.

Ludwig 906.

Lübbert 957 .

Müller, H. G. 882. Plauchu 924.

Münzel 895. Pleasonton 804.

Murmann 828. Plumlee 880.

N. V. de Bataafsche Plyler 806 .

Petroleum Mij. Pokrowski 925.

918. 933. 935. 955. Polatnick 840.

N. V. Onderzoekings- Polikarpowa 919. instituut, ,Rese- Pollard 824 arch" 949. Polonsky 861. 862.

N. V. Philips' Gloei- Pope 880 .

lampenfabrieken Porai-Kcschitz 855. Salow 810.

Post 830 .

gister Co. 960.

Naumow 906.

Nawrocki 884 .

Nelson 824.

Neunzig 921.

Newman 807 .

Nicholson jr. 880

Nicolay Titlestad

Corp. 953.

Nielsen 897.

Nilsson 806 .

Nitralloy Corp. 922.

Nola, di 884.

Nordlander 945 .

Nordstig 896.

Norrish 834.

Nottes 951 .

Novellie 902.

Nowak 938.

Elektrowerk

G. m. b. H.

Ramakrishnan 941.

Nußbaum, A. L. 865. Rappaport 88

Nutting 873 .

Nygard 804

Ostener 827.

Reich 891.

Reich 891.

rikanische Magne- Rene 882

sit Akt.-Ges. 914.

Ostholm 896.

Offenbacher 809.

Oldham 876.

Oliaro 884.

Omodei-Zorini 884.

O'Neill 917.

Orth 917.

Orton 890

Osmond 812.

Oulu Osakeyhtio 945. Ritow 809. 


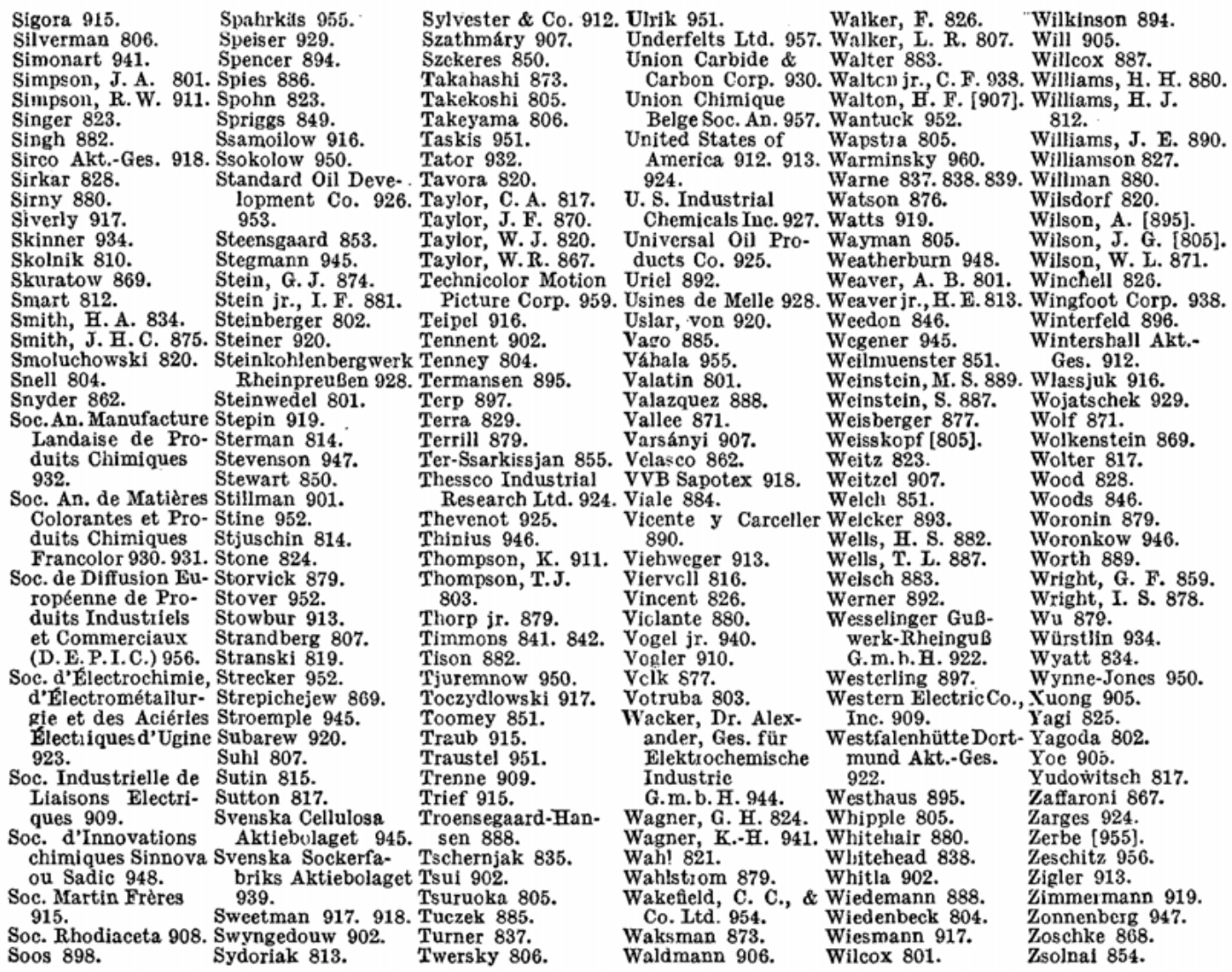

\title{
CHEMISCHES ZENTRALBLATT
}

In Kürze erfcheint:

\section{Ergänzungsband 1949}

\author{
Preis DM 60.-- \\ Wir weifen darauf hin, daf die Lieferung des Ergänzungs* \\ bandes nicht innerhalb des Abonnements vorgenommen wird.
}

